\title{
Politisch korrekt von der Wiege bis zur Bahre
}

\author{
Eine Initiative des englischen Ge- \\ sundheitsministeriums zeigt, dass \\ man es mit der politischen Korrekt- \\ heit auch übertreiben kann.
}

— Im Jahr 2005 veröffentlichte das englische Gesundheitsministerium ein Dokument mit dem Titel „Beseitigung der gemischt geschlechtlichen Unterbringung in Krankenhäusern“. Diese Verordnung zielte darauf ab, die Würde und die Privatsphäre aller Patienten während ihres Krankenhausaufenthaltes zu wahren. Eine Pressemitteilung des Ministeriums aus dem Jahr 2010 konstatierte, dass immer noch Zehntausende von Patienten im
Krankenhaus gemischt geschlechtlich untergebracht seien. Im Januar 2011 kündigte die Gesundheitsministerin an, dass allen Organisationen des öffentlichen Gesundheitsdienstes, die gegen diesen Kodex verstoßen, mit finanziellen Sanktionen gedroht werde.

Während einer Visite auf der Frühgeborenenstation fiel Ärzten in einem Kinderkrankenhaus ein Schild mit folgender Inschrift auf:

„Bitte beachten Sie, dass es unsere Politik auf

Schild auf der Neugeborenen-Station. der Neugeborenen-Station ist, Jungen und Mädchen in nebeneinander stehenden Bettchen zu betreuen.

Sollten Sie mit diesem Vorgehen nicht einverstanden sein, bitten wir Sie, diesen Umstand mit der diensthabenden Schwester zu besprechen."

Es ist erstaunlich, welche Blüten der politischen Korrektheit die Furcht vor ökonomischen Konsequenzen in Krankenhäusern hervorbringen kann. Ob die Verordnung wohl auch für die Leichenkammern gilt?

- M.Green, M. Davison

(Korres.:mftgreen@doctors.org.uk): Political correctness: a step too far? Brit. Med. J. 2011; 343: d7451.

\section{Wenn zwei sich mögen, gähnen sie gemeinsam}

\section{Ansteckendes Gähnen wird vorwie- gend durch die emotionale Nähe zwischen Personen gesteuert und nicht durch Variablen wie Geschlecht oder Nationalität.}

— Sicher haben Sie an sich selbst schon öfter bemerkt, dass Gähnen von Menschen in einer Gruppe ansteckend wirkt. Psychologische Untersuchungen zeigen, dass dieses Phänomen erst in einem Alter ab vier bis fünf Jahren auftritt, wenn Kinder anfangen, die Emotionen anderer Personen wahrzunehmen. Bei Menschen mit empathischen Störungen, zum Beispiel Autisten, wird ansteckendes Gähnen deutlich seltener beobachtet, bei Personen mit in der Selbsteinschätzung ausgeprägter Empathie tritt es mit größerer Wahrscheinlichkeit auf.

In einer unbeeinflussten Beobachtungsstudie wurden 109 Erwachsene in verschiedenen Ländern und Erdteilen in ihrer natürlichen Lebens- und Arbeitsumgebung über ein Jahr hinweg in Zeitperioden zwischen sechs Minuten und zwei Stunden beobachtet. Sobald ein Proband gähnte (der Auslöser) wurden alle Gähnepisoden der Personen registriert, die mit dem Auslöser im Abstand von höchstens drei Metern in akustischem oder optischem Kontakt standen. Die sozialen Bezüge zwischen dem Auslöser und den Gähn-Nachahmern wurden durch intensive Befragungen evaluiert.

Ansteckendes Gähnen kann bis zu 5 min nach dem Auslöser hervorgerufen werden mit einem Gipfel von etwa 3 min nach dem Auslöser. Es zeigte sich, dass nur enge soziale Bezüge als Vorhersageparameter für das Auftreten, die Häufigkeit und die Latenz des ansteckenden Gähnens gewertet werden konnten. Die Wahrscheinlichkeit des ansteckenden Gähnens war am größten bei leiblichen Verwandten, dann bei engen Freunden, am geringsten bei entfernten Bekannten und Freunden. Damit fällt das ansteckende Gähnen in die Kategorie anderer Parameter der Empathie, die ebenfalls bei leiblichen Verwandten stärker ausgeprägt sind als bei sonstigen Mitmenschen. Obwohl Frauen grundsätzlich eine höhere Empathie nachgesagt wird, spielten Parameter wie Geschlecht oder Nationalität keine Rolle für die Häufigkeit des ansteckenden Gähnens.

\section{Kommentar}

Vielleicht eignen sich die Ergebnisse dieser Untersuchung ja gut für das Kennenlernen eines neuen Arbeitsumfeldes. Wenn Sie demnächst in der Konferenz ein Gähnen nicht unterdrücken können, beobachten Sie doch mal die Kollegen. Wer innerhalb von fünf Minuten ebenfalls gähnt, ist Ihnen vielleicht am meisten gewogen. Oft dauert es viel länger, bis man dahinter kommt, wer es gut mit einem meint.

\footnotetext{
- I.Norscia, I. E. Palagi

(Korres.: betta.palagi@museo.unip.it) Yawn contagion and empathy in homo sapiens. PLoS one $2011 ; 6(12)$ : e28472
} 